\title{
An optically coupled power stimulus isolation unit with high voltage and fast rise time output
}

R.O. Brasil and J.H. Leal-Cardoso
Departamento de Ciências Fisiológicas, Centro de Ciências da Saúde, Fundação U niversidade Estadual do Ceará, Fortaleza, CE, Brasil

\section{Correspondence \\ J.H. Leal-Cardoso \\ Departamento de Ciências Fisiológicas \\ Centro de Ciências da Saúde \\ Fundação Universidade Estadual \\ do Ceará \\ 60740-000 Fortaleza, CE \\ Brasil \\ Fax: $+55-85-292-4299$}

Received June 1, 1998

Accepted February 19, 1999

\section{Abstract}

Recent technological developments have created new devices that could improve and simplify the construction of stimulus isolators. HEXFET transistors can switch large currents and hundreds of volts in nanoseconds. The newer opto-isolators can give a pulse rise time of a few nanoseconds, with output compatible with MOSFET devices, in which delays are reduced to nanoseconds. Integrated DC/DC converters are now available. Using these new resources we developed a new electrical stimulus isolator circuit with selectable constant-current and constant-voltage modes, which are precise and easy to construct. The circuit works like a regulated power supply in both modes with output switched to zero or to free mode through an opto-isolator device. The isolator analyses showed good practical performance. The output to ground resistance was $10^{11} \mathrm{ohms}$ and capacitance 35 picofarads. The rise time and fall time were identical $(5 \mu \mathrm{s})$ and constant. The selectable voltage or current output mode made it very convenient to use. The current mode, with higher output resistance values in low current ranges, permits intracellular stimulation even with tip resistances close to 100 megaohms. The high compliance of $200 \mathrm{~V}$ guarantees the value of the current stimulus. The very low output resistance in the voltage mode made the device highly suitable for extracellular stimulation with low impedance electrodes. Most importantly, these characteristics were achieved with a circuit that was easy to build and modify and assembled with components available in Brazil.

We have described a stimulus isolator (1) with high voltage $(\leq 150 \mathrm{~V})$ and current $(\leq 5$ $\mathrm{mA}$ ) output using bipolar transistors and components available in Brazil. In order to obtain a design with the power required by the experimental protocol, the output circuit consisted of interchangeable voltage- and current-regulated sources. In this isolator the low output resistance of the regulated voltage mode assures the assigned pulse voltage amplitude, which is difficult to measure in isolated systems. We achieved current regu-

\section{Key words}

- Electrical isolation

- Electrical stimulation

- Pulse stimulation

- Stimulus isolator lation for the current-regulated mode by increasing the isolator output resistance through current sampling and feedback.

The performance of this isolator was considered to be good. However, despite some advantages over similar commercially available isolators, it presented some limitations common to the other isolators, such as the optical coupling delay and limitations in frequency response due to power transistors.

Since the development of our stimulus isolator, technological developments have 
led to new devices that have improved and simplified this apparatus. HEXFET are MOSFET transistors developed by the electronic industry to switch large currents and hundreds of volts in nanoseconds. The HEXFET is also appropriate for linear applications. The need for faster data transmission brought about the development of optoisolator devices operating in the $\mathrm{MBd}$ range (more than one million bits per second), which implies a pulse rise time of a few nanoseconds with output also compatible with the input of MOSFET devices. Thus, opto-isolator delays were reduced to nonsignificant values (nanoseconds) when compared with fast electrophysiological phenomena such as neuronal action potentials. DC/ DC converters with $0.5-\mathrm{W}$ output and $12-\mathrm{V}$ input later became available. Using these new resources and maintaining the basic circuit idea of the former stimulus isolator (selectable constant-current (CC) and constant-voltage (CV) mode with relatively large power outputs) and the same goal (a precise and easy to build circuit with components available in Brazil) we developed a new circuit whose description follows.

Figure 1 shows the complete electronic circuit. Eight DC/DC converters with $12-\mathrm{V}$ inputs are connected in parallel to a battery. The 24-V outputs and the battery are connected in series to produce a $204-\mathrm{V}$ total output. The circuit without the Q2 and Q3 transistors is a conventional regulated-voltage power supply. In the absence of an input, Q2 transistor conducts with the on-state resistance close to $5 \Omega$, then its drain voltage to reference goes close to zero, turning off Q1. When a pulse is applied to the isolator input, the optically coupled signal turns off Q2 and this releases Q1, setting a voltage output which is determined by the position of the cursor in P1, less the small and practically constant gate/source polarization of Q1.

The scales of voltage, 5 to 50,10 to 100 and 20 to $200 \mathrm{~V}$, can be changed by switching S1, taking advantage of the fact that the pick-up of the converters is adjusted in the P1 potentiometer.

When the input pulse ends, Q2 conducts again and this turns off Q1, ending the output pulse and discharging the capacitances associated with output through D1 and Q2.

The output pulse rise time is dependent on the time constant determined by the total Q2 drain/source capacitance and R1 resistance, since P1 plus R2 is much higher than $\mathrm{R} 1$. With a small $\mathrm{R} 1$, the rise time decreases at the expense of the power consumption. We adjusted $\mathrm{R} 1$ to a $5-\mu$ s rise time, which is $0.5 \%$ of the period of the fastest train of stimulation of practical importance on electrophysiology.

The Q3 circuit is a linear amplifier configured to work as a current stabilizer. It controls the output voltage to keep the level of current adjusted in the $\mathrm{P} 2$ potentiometer constant. This, in addition to permitting the isolator to operate in the current mode, protects against output short circuits.

The isolator offers two ranges of current selected at the S3 switch. With S3 in the down position the range is from 50 to 500 $\mu \mathrm{A}$. In the up position (see Figure 1) the range is from 0.5 to $5.0 \mathrm{~mA}$.

The modes of CC, automatic (AUT) and $\mathrm{CV}$ are options in the switch S2-1/S2-2. In the automatic and voltage mode the gate of Q3 is associated with the central lead of the P1 potentiometer, allowing adjustment of the voltage output. The output current goes through R6, a fixed resistor, in the voltage mode, to protect against short circuits in output and permit more power for the load.

In the current and automatic modes, S2-2 enables the selection and adjustment of current ranges. Only in the current mode does S2-1 connect the Q1 gate to the P1 top, keeping this voltage fixed to define the source current compliance. Compliance, or the maximum value of output voltage in current mode, can be chosen in S1. Occasionally it becomes necessary to protect the preparations from high voltage. 
All the controls are active in the automatic mode. The experimenter may adjust either voltage or current according to his requirements.

This isolator was designed to permit the use of a larger number of DC/DC converters with serial output connection (so as to increase the maximum voltage output), without the necessity of large circuit modification. These isolators may be connected with inputs in parallel or in different sources and the outputs may be connected in series to increase the voltage output or to shape waveforms.

Although the factory parameters of the power transistors are not appropriate for this type of analysis (since they were intended for much larger currents) we will use them to evaluate the performance of the isolator (2).

The output resistance in the voltage mode, when Q3 is not active because the load current is not enough to keep it polarized, works like a non-stabilized voltage regulator. The output resistance of the source follower is reduced to the inverse of transconductance (3), so that:

$$
\mathrm{R}_{\mathrm{o}} \approx 1 / \mathrm{g}_{\mathrm{fs}}=1 \Omega
$$

This very low resistance makes the isolator output closely approach a constant voltage source, considering the much higher loads connected to the isolator output. The resistor structure for current control connected in series to the load taking the output resistance to the $1 \mathrm{k} \Omega$ range is still satisfactory. In the case of no voltage feedback, reference voltage (voltage at P1 center tap) will directly reach output. In the last resource it is important that the voltage stability of the converters maintains the assigned output voltage in

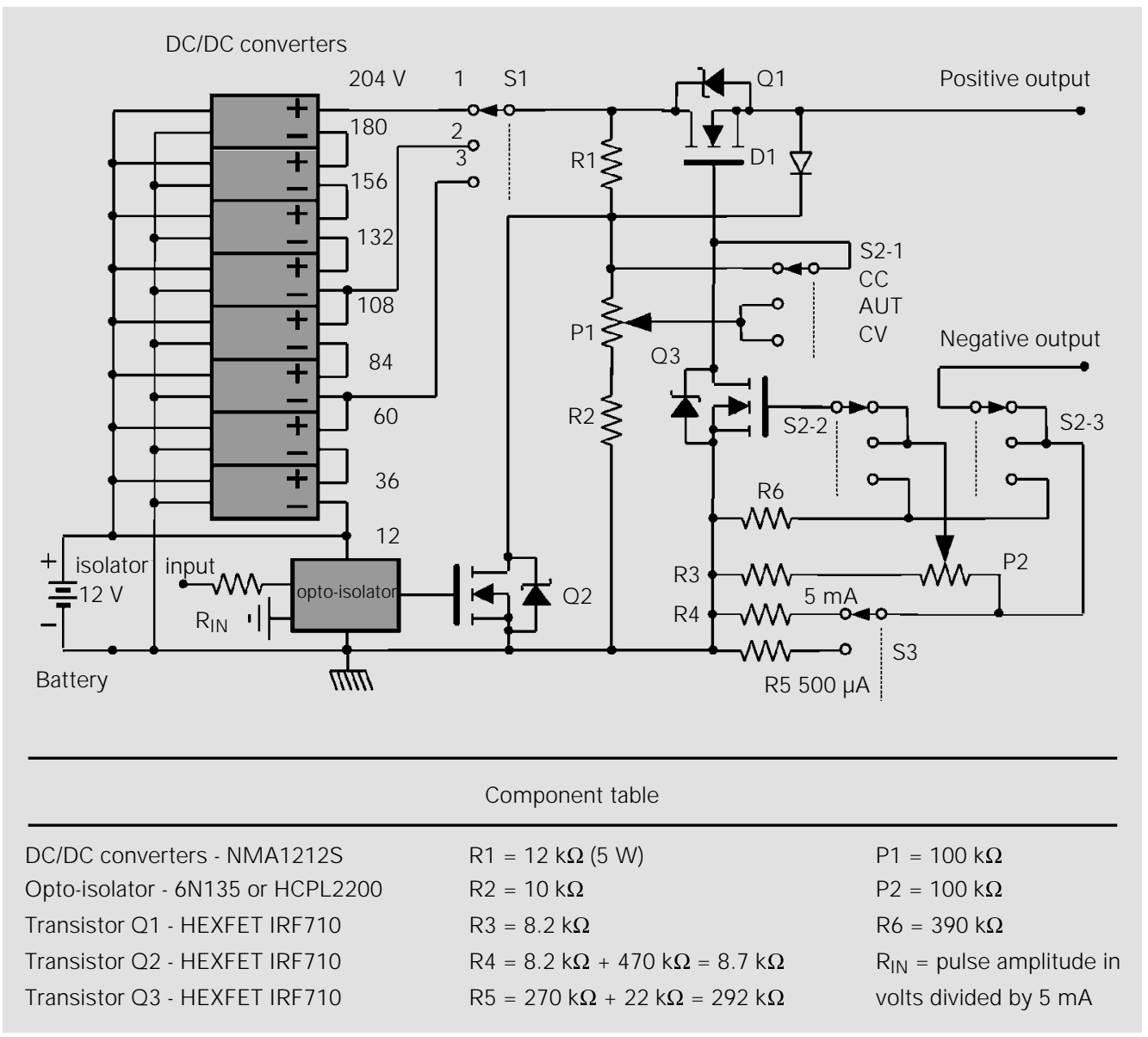

Figure 1 - HEXFET power stimulus isolator circuit. S1, position 1: 20 to $200 \mathrm{~V}$; position 2: 10 to $100 \mathrm{~V}$; position 3: 5 to $50 \mathrm{~V}$. S3, position 1: 0.5 to $5 \mathrm{~mA}$; position 2: 50 to $500 \mu \mathrm{A}$. CC, Constant current; AUT, automatic; CV, constant voltage. 
all the current range of the isolator. With pulse output low, in the worst case, the current drain does not reach half of the nominal converter output current.

In the constant current mode, with the Q3 transistor amplifier working, the feedback loop is closed. In this condition the feedback loop multiplies the output resistance by the desensitization factor, so that:

$$
R_{0}=(1+B \cdot A)
$$

where $B=1$ and $A=g_{f s} \cdot R_{L}(Q 3$ amplifier gain).

With $0.5 \mathrm{~mA}$ output, $\mathrm{R}_{\mathrm{o}}$ without feedback is close to $8 \mathrm{k} \Omega$ and the effective $R_{\mathrm{L}}$ is $\mathrm{R} 1(12 \mathrm{k} \Omega$ ), and thus the output resistance value should be $R_{\text {of }} \cong \beta \cdot A \cdot R_{0}=1 \times 1.2 \times 10^{4}$ $\times 8 \times 10^{3} \cong 100 \mathrm{M} \Omega$. In the other range the value increases 10 times, permitting output resistances of $1000 \mathrm{M} \Omega$, which can keep current approximately constant with 100$\mathrm{M} \Omega$ intracellular microelectrodes.

The isolator performance parameters are suitable for intracellular and extracellular electrophysiological stimulation $(4,5)$ and comparable to other commercially available stimulus isolation units $(6,7)$.

To test a prototype of the stimulus isolator, after connecting the two grounds, shown in the figure connected to the opto-isolator, the procedure described below should be followed.

Start with no voltage connected to the input of the opto-isolator, measuring the DC voltages at the three positions of the S1 switch with a multimeter. This measurement indicates the state of the DC/DC converters. If the expected voltages (204, 108 and $60 \mathrm{~V}$ ) are not found, check the converters individually. The voltage at the center tap of the P1 potentiometer as well as at the positive output should be close to zero, when as referred to ground, independent of any external control. The opto-isolator output should be $4 \mathrm{~V}$ above ground and the drain voltage from Q1 to ground should be close to zero.

Connect a positive DC source to the opto- isolator input with a voltage which should be $\mathrm{R}_{\mathrm{IN}} \cdot 5 \mathrm{~mA}$ as specified in the component table in the figure, and the coupler output should be close to zero. In the CV mode measure the voltage variation from P1 center tap and from the positive output, as referred to ground for all the three output voltage ranges. Check if the output voltage ranges are correct and the voltage between them and the center tap of P1 are set close to $4 \mathrm{~V}$.

A static test of the control of current may be carried out. At the higher voltage range, with the $\mathrm{P} 1$ potentiometer set to the maximum output and in the CC mode, set the range to $5 \mathrm{~mA}$. With a $10 \mathrm{k} \Omega(1 / 2 \mathrm{~W})$ load resistor, check the voltage output variation from 50 to $100 \mathrm{~V}$, acting on $\mathrm{P} 2$ potentiometer. With another resistor in series increase the load to $20 \mathrm{k} \Omega$ and repeat the check. The measured voltage values should now double because the current range is maintained. Set the two resistors in parallel and the measure should drop to half. Using $100-\mathrm{k} \Omega$ resistors, repeat the same procedure for the 500 $\mu \mathrm{A}$ range. The measurements should be repeated.

In the $\mathrm{CV}$ mode with a $10-\mathrm{k} \Omega$ load the maximum voltage adjustment should be limited to approximately $100 \mathrm{~V}$, since R6 limits the current to $10 \mathrm{~mA}$ to protect against output short circuits.

The dynamic test of the stimulus isolator should be done using a two-channel oscilloscope to watch pulses of the isolator input and output. The generator coupled to the input of the isolator for the test must drive a pulse current amplitude larger than $5 \mathrm{~mA}$ necessary for the optical transfer. One should measure on the trailing and ending edges of the output waveform (the rise- and falltime, i.e., the time elapsed in the variation of voltage from 10 to $90 \%$ of the steady-state final value). The delay of the output can be measured by synchronizing the oscilloscope with the stimulator input waveform and looking for time difference in the output waveform. 


\section{References}

1. Brasil RO \& Leal-Cardoso J H (1986). Isolador de estímulo para uso em eletrofisiologia. Resumos da I Reunião Anual da Federação das Sociedades de Biologia Experimental, São Paulo, SP, August, 309.

2. Horowitz H (1989). The Art of Electronics. Cambridge University Press, New York.
3. Millman J \& Grabel A (1987). Microelectronics. McGraw Hill Inc., New York.

4. Brown PB, Maxfield BW \& Moraff $\mathrm{H}$ (1973). Electronics for Neurobiologists. Massachusetts Institute of Technology, Cambridge, MA.

5. Strong P (1970). Biophysical Measure- ments. Tektronics Inc., Beaverton, OR.

6. World Precision Instruments (1998). International Laboratory Equipment Sale Catalog. Sarasota, FL.

7. Axon Instruments Inc. (1998). Product Catalog. Foster City, CA. 